\title{
Intra-Atomic Gravitational Shielding (Lensing), Nuclear Forces and Radioactivity
}

\author{
Robert A. Sizov* \\ Solid State Physics Institute, Moscow, Russia \\ Email: sizov.robert@gmail.com
}

How to cite this paper: Sizov, R.A. (2019) Intra-Atomic Gravitational Shielding (Lensing), Nuclear Forces and Radioactivity. Journal of Modern Physics, 10, 59-73. https://doi.org/10.4236/jmp.2019.101006

Received: December 13, 2018

Accepted: January 22, 2019

Published: January 25, 2019

Copyright $\odot 2019$ by author(s) and Scientific Research Publishing Inc. This work is licensed under the Creative Commons Attribution International License (CC BY 4.0).

http://creativecommons.org/licenses/by/4.0/

\begin{abstract}
The discovery by the author of real magnetic charges and true anti-electrons in the atomic structures allowed him to establish that the gravitational field (GF) in reality is the vortex electromagnetic field. Depending on the vector conditions the gravitational fields can be either paragravitational (PGF) or ferrogravitational (FGF). Masses (atoms, nucleons, etc.) emitting PGF manifest so-called attraction to each other. In fact, this process is the pressing of atoms or nucleons to each other by the forces of gravitational "Dark energy". Namely the gravitational "Dark energy" which is formed between the masses emitting PGF and compressing of nucleons in atomic nuclei is the main force factor determining the formation of nuclear forces. Masses that emit FGF are repelled from PGF sources, for example, from the Earth. The last gravitational manifestation, discovered by the author, this is of the effect of the gravitational levitation. The atomic shell and atomic nucleus are autonomous sources of gravitational field in atomic compositions. The gravitational fields emitted these sources, by its physical parameters, are different gravitational fields, what associated with differences in the magnitudes charges of magnetic and electric particles in their compositions. The noted differences in the parameters of the GF are of reason that in atoms the process of extrusion of foreign gravitational field from the region of given gravitational source is realized. This effect should be called the effect of intra-atomic gravitational shielding (IAGS). Within the framework of this effect the shell of the atom is a kind of gravitational "insulator" that prevents the PGF of the nucleons from leaving beyond of the atom. As result of the IAGS effect, the concentration PGF of nucleons is realized only in the region of the nucleus, which leads to an increase in nuclear forces. However, the resistance of the marked "insulator" is finite and if the critical voltage PGF on the nucleus is exceeded, the complete shielding of the nucleon fields by the atomic shell is broken. As
\end{abstract}

*The author is the doctor of physical and mathematical sciences. At present works as individual researcher. 
result of the leakage of a part of the PGF of nucleons beyond the atom, the density of this field in the region of the nucleus decreases significantly, which leads to a weakening of the nuclear forces and often leads to radioactivity. The effect of gravitational shielding is directly related to such a well-known concept as the mass defect of the nucleus. It is the exclusion of the gravitational field formed by the nucleons in the composition of the atomic nucleus as a result of the full IAGS effect that creates the illusion of atomic mass defect.

\section{Keywords}

Magnetic Charges, Antielectrons, Magnetons, Antimagnetons, Bispinors, Physical Mass, Negative Masses, Vortex Electromagnetic (Gravitational) Field, Paragravitation and Ferrogravitation, "Dark Energy", Gravitational Shielding (Lensing), Defect of Mass, Nuclear Forces

\section{Introduction in Real Physics of Magnetic and Electric Charges, Gravitational Field and "Dark Energy"}

Experimental and theoretical studies of the author (the period from 1968 to the present time) have shown that magnetic spinor particles (magnetic charges) are real structural components of atoms and substance and are the direct sources of all magnetic fields in Nature [1] [2]. It should note that initial experiments which prompted the author to studies' problem of participation magnetic charges in the structures of atoms and substance were his experiments with magnetic scattering of neutrons in the ferrimagnetic crystals [3]. In addition, the author's research has allowed establishing that in atoms and substance as the structural components "work" and such of real Antimatter particles as true anti-electrons with charge $\mathrm{e}^{+}$. These last particles are neither positrons nor Dirac's "holes". The real particles, noted above, for more than hundred years are not recognized by official physical science. The introduction of these particles into the basic physical concepts significantly changes the interpretation of a huge number of physical effects, and also allows us to discover new physical manifestations and patterns.

\subsection{Magnetic and Electrical Charges in Structures of Atomic and Nucleonic Shells}

The results of the author's research showed that the shells of atoms are electromagnetic, and not electronic as commonly believed. Magnetic and electric charges forming atomic shells exist in the compositions of corresponding dipoles circulating on atomic orbits. The number of magnetic charges in the compositions of shells is approximately equal to the number of electric charges. The values of charges of the spinor particles in atomic shells correspond to the condition $\mathrm{e}=\mathrm{g}$, where $\mathrm{g}$ is the charge of magnetic particle. Magnetic dipoles consist of 
magnetons, i.e. material magnetic particles with charge $\mathrm{g}^{-}$and antimagnetons, i.e. particles Antimatter with charge $\mathrm{g}^{+}$. These magnetic particles are magnetic analogs of electrons and antielectrons. The magnetic charges presented above emit magnetic fields exclusively because of their magnetic nature which has nothing to do with electrical particles. In other words, magnetism and electricity are physically independent natural manifestations [4].

It is important to note here that the first person who observed real magnetic charges (in his opinion, magnetic ions) was Felix Ehrenhaft, who made his remarkable discoveries at beginning of the $20^{\text {th }}$ century [5]. The reasons which, more than one hundred years, inhibit the recognition of valid conclusions of F. Ehrenhaft were discussed in detail in the publications of the author (see, for example, [2]).

It should be noted such interesting topic of the theoretical physics as magnetic monopoles. Under the concept "magnetic monopole" is commonly understood as theoretical electrified structures that are designed to create a magnetic field. The basis for the design of these structures, as a rule, is the theory of Dirac's magnetic monopole [6]. However, this monopole is capable of emitting exclusively the vortex magnetic field, which is described by the vortex vector $\operatorname{rot} H$ [7]. For this reason the Dirac monopole and its derivatives cannot have any relation to real magnetic poles emitting the polar magnetic field, natural to them, with field strength $H$. By their physical nature real magnetic charges are magnetic analogues of electrons, as well as other fundamental electrical particles. Given the physical irreducibility of real magnetic charges and theoretical magnetic monopoles, the author considers it pointless to draw any logical Parallels between them.

In atomic structures, in composition of electric dipoles, there are true antielectrons, i.e. of real Antimatter particles with charge $\mathrm{e}^{+}$. Note, that these antielectrons no direct relation to the positrons and has nothing to do with the so-called electron vacancies or Dirac "holes" [8].

The author in the publication [9] showed that nucleons (proton and neutron) are of the atomic-shaped electromagnetic structures, i.e. like of atoms include the nucleon shell and nucleus. The magnitude of the electric and magnetic charges in the nucleon shells are, according to the author's research, 1/2 or less of the magnitude charges in atomic shells.

It is important to note that the basic (unperturbed) state of the spinor particles is the state of bispinor. In this state the charges in pairs + and - are tightly pressed together by the forces of "Dark energy" what leads to compensation of the external activity of their spins. Such pair does not manifest itself by external spinor field and, in practice, is not detected. The process of dense compression of a particle and an antiparticle described above and the exclusion of this pair from the information sphere provoked the emergence of such a fictitious concept as the annihilation of particles in the existing physical theory. This concept was formed on basis of observed interaction of electron and positron, as result of which only gamma quanta are detected. The positron is not is elementary spinor particle but is the varieties of mass, that is, it is the electromagnetic 
structure atomic-shaped form. A true antielectron "sits" in the positron structure as its nucleus. Upon contact of electron and positron the electron connect with the antielectron, i.e. that is, with the positron nucleus with the formation of bispinor. As for the electromagnetic (quark) shell of the positron, it actually annihilates, and the evidence for its destruction is the observed gamma quanta. At the same time, the main protagonists of the so-called annihilation of particles (electron and true antielectron) do not annihilate, but remain unchanged in the composition bispinor.

\subsection{The Electromagnetic Vortex (Gravitational) Fields and Essence of Physical Mass}

The author's research has shown that electric and magnetic charges forming atomic and nucleonic shells exist in the compositions of the corresponding dipoles which rotate on atomic and nucleonic orbits. Namely the electromagnetic shells of atoms are the natural sources (generators) of gravitational field (GF) which, in reality, is of vortex electromagnetic field [10]. The elementary source of gravitational field is the spinor electromagnetic quasi-particle which received the name S-Graviton (S from "source"). The composition of S-Graviton: two spinors (electric and magnetic) and two antispinor corresponding to them. The S-Graviton is combination of the electric and magnetic dipole rotating in antiphase at same atomic or nucleonic orbit. The model representation of the atomic orbital electromagnetic current or S-Graviton can be written in following form: $\operatorname{rot}\left[\boldsymbol{J}_{e}-\boldsymbol{J}_{g}\right]$ where $\boldsymbol{J}_{e}$ and $\boldsymbol{J}_{g}$ are vectors of density instantaneous currents of electric (e) and magnetic (g) charges in corresponding of orbital flows. Then the equation process gravitational field formation by means the atomic S-Graviton can be presented in the form: $k \operatorname{rot}\left[\boldsymbol{J}_{e}-\boldsymbol{J}_{g}\right]=\operatorname{rot}[\boldsymbol{E}-\boldsymbol{H}]$ where $\boldsymbol{E}$ and $\boldsymbol{H}$ are vectors of the instantaneous electric and magnetic field strength in structure of the vortex electromagnetic (gravitational) field, $k$ is the proportionality coefficient.

If the polarization of vortex vectors $\operatorname{rot}\left[\boldsymbol{J}_{e}-\boldsymbol{J}_{g}\right]$ of S-Gravitons is realized in structures of Physical masses (in atoms, nucleons, substance et al.) that is accompanied by polarization corresponding of vortex vectors $\operatorname{rot}[\boldsymbol{E}-\boldsymbol{H}]$, then by analogy with magnetic fields of the ferromagnetics, the gravitational fields being emitted by these masses can be called the ferrogravitational fields (FGF). The gravitational fields formed by masses in the absence polarization of the vortex vectors of S-Gravitons in their compositions are tensor or quasi-scalar fields. And again, by outward analogy with magnetism, such fields can to define as paragravitational fields (PGF).

The mathematical expression corresponding to the states of FGF is have the form $\langle\operatorname{rot}[\boldsymbol{E}-\boldsymbol{H}]\rangle \neq 0$. The gravitational fields corresponding to the condition: $\langle\operatorname{rot}[\boldsymbol{E}-\boldsymbol{H}]\rangle=0$, by analogy with the paramagnetism can be defined as paragravitational field.

The physical masses which emit the ferrogravitational fields will push off from 
masses-sources of the paragravitational fields, for example, from Earth. This last effect discovered by the author of the article called as the effect Gravitational levitation [11]. It is important to note that the masses emitting FGP are so-called negative masses. The well-known representative of the "negative mass" is the atom of ordinary hydrogen or protium.

In his publications, since 2001, author tried to explain that all varieties of Physical Mass are the electromagnetic structures of atomic-shaped type consisting of electric and magnetic spinor particles. Main characteristic property of all varieties of mass is ability to emit the gravitational field that is formed as the result joint orbital currents of electric and magnetic charges and is the vortex electromagnetic field. In addition, the author showed that the formation of photons also falls, exclusively, within the competence of mass [12].

It is necessary to emphasize that Physical Mass and, for example, Matter are completely different physical categories. By the Physical masses, for example, are atoms, nucleons, substance. It is especially important to note that the individual spinor particles as electric, so and magnetic are massless, because mass is the result their joint structural union. For example, the electron is massless particle and no "divine bosons" can't give it mass.

\section{3. "Dark Energy" Is Global Force Factor That Is Responsible for All the So-Called Interactions between Masses and Charges}

The results research of real magnetic charges allowed the author to formulate the conception of the World Physical Triad (PT) according which the real World consists of three fundamental phases: of the Matter, Antimatter and Energo-phase (Energo-medium) [13] [14].

The Phase of Matter is inhabited with magnetic and electric spinor particles with negative charge, and the Antimatter is formed by electric and magnetic particles with positive charge. Particles Antimatter constitute half all of real spinor particles, i.e. charged particles in real World, and their absence in the physical representations is determined by Physics their of confinement in the atoms and substance which is radically different from the confinement, for example, of electrons.

In the basic (undisturbed) state the Energo-phase (Energo-medium) is the isotropic high-density gas-like (possibly also quasi-fluid) medium formed by its own fundamental particles referred to as the energions which are spinless and massless. These particles are very small, they move in all directions at speeds close to the speed of light and can only be of two types: the Left and Right what linked with the appropriate direction of their own rotation. Super-high mobility and not-inertial behavior of the energions allow the particles and masses to move relatively freely in Energo-medium when this medium is in basic (undisturbed) state.

According to the Physical Triad Concept all forces direct action on the par- 
ticles and masses, which are implemented in the real World are the forces of the so-called "Dark energy" ("DE") which are determined by non-equilibrium states in the "Energo-medium" in the form of oblasts of local pressures $P_{\varepsilon}$ created by energions $(\varepsilon)$. The formation of "Dark Energy" in Energo-medium is induced by spinor fields, i.e. fields of charged particles. All variety of spinor fields, including gravitational fields, don't have any real of power significance. They only play the role of intermediaries exerting influence on state of the Energo-medium and inducing formation of "Dark Energy" in it. Namely "Dark Energy" is real forces factor, performing the dynamics and the so-called interactions of masses and charged particles as in the scale of the Universe (the movement of galaxies, stars, planets and other objects) so and in the microcosm, for example, the dynamics of the spinor particles within a Physical masses of such as in atoms, nucleons, etc.

The gravitational fields radiated by masses (bodies) create in the area between them zones of negative or positive "Dark energy". Forces of negative "DE" $\left(\boldsymbol{F}_{D E}^{-}\right)$ which realized between masses emitting the paragravitational field is determines all physical manifestations within the framework of the law of Universal Gravitation. As for the positive "DE", so it is formed between the masses emitting PGF and FGF. The forces of the positive "DE" repel bodies from each other and are responsible for such an important effect as gravitational levitation.

The "Dark energy" realized in practice can associate with spinor fields which formed its, and therefore you can define the "Dark energy", for example, as the gravitational "DE" or electrostatic "DE". Considering that the gravitational field is the vortex electromagnetic, i.e. the spinor field, for the present study it is important to find out the mechanisms formation of the gravitational "Dark energy" and the features of it effect on the masses (bodies).

\section{Gravitational Shielding in Hydrogen Atoms}

Section 1.2 of this article showed that the gravitational fields of the masses (atoms, nucleons, etc.), in depending on the ordering of the vortex vectors $\operatorname{rot}[\boldsymbol{E}-\boldsymbol{H}]$ in the compositions of the gravitational fields (GF) emitted by them, can be both paragravitational (PGF) and ferrogravitational (FGF). The classical source of GF is the electromagnetic quasiparticle consisting of two coupled dipoles (electric and magnetic) rotating in one atomic or nucleon orbit. S-Graviton is the shell of all hydrogen atoms (protium, deuterium and tritium).

Diagram of structure of the atom so-called lightweight hydrogen (protium) is shown in Figure 1. Recall that the atomic shells of all elements, like the shell of hydrogen atoms, are electromagnetic, and not electronic, as is now commonly believed. The magnitudes of the charges of the electric and magnetic spinor particles constituting the atomic shells of all the elements correspond to the condition $\mathrm{e}=\mathrm{g}$. The considering that S-Graviton is an elementary source of a gravitational field, the shell of a hydrogen atom emits namely FGF. As for the proton $\left(p^{+}\right)$, which is located in the center of the atom and performs the function of the nucleus, it emits a PGF. It is known that the proton is attracted, more precisely, 


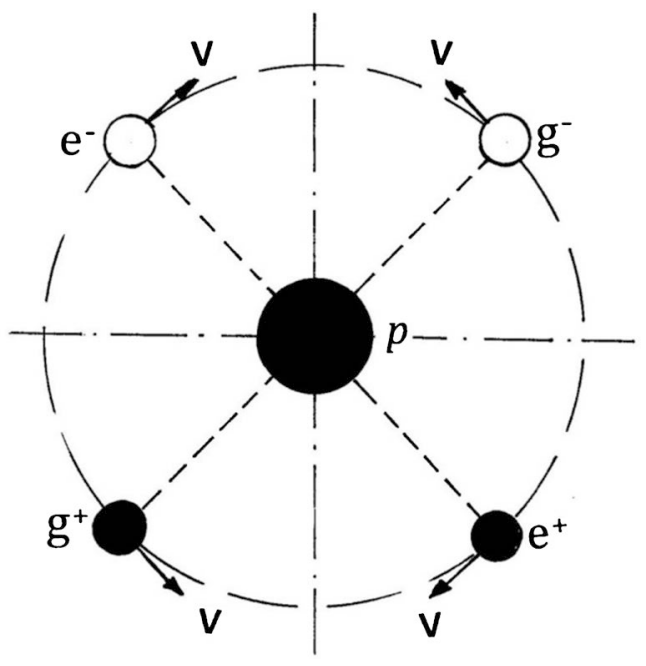

Figure 1. Scheme the electromagnetic device of atom protium. Through white circles in the Figure 1 are shown of negative charged electric and magnetic spinors: electron $\left(\mathrm{e}^{-}\right)$ and magneton $\left(\mathrm{g}^{-}\right)$, and black circles denote of positively charged antispinors corresponding to them with charges $\mathrm{e}^{+}$and $\mathrm{g}^{+}$. The vectors instantaneous velocity $\boldsymbol{V}$ is indicate the direction rotation of the magnetic and electrical dipoles in the same orbital of the hydrogen atom.

pressed to the Earth by the forces of the gravitational "DE", what is unequivocal sign of its PGF.

In the composition of the atom protium is coexist two autonomous sources of GP: shell emitting FGF and nucleus-proton emitting PGF. However, in gravitational interactions, protium manifests itself as the source of FGF, which, we recall, is generated by its EM-shell. In favor of the latter statement is testifies by the known volatility of protium which is determined by the effect of gravitational levitation discovered and studied by the author.

The most probable cause that does not allow proton PGF to go beyond the atomic shell of the protium atom and manifest itself in gravitational interactions is the effect of intra-atomic gravitational lensing (IAGL). Through the term "gravitational lensing" in physics all types of deviations by masses of EM-fields are defined. Within this broad theme its physical subsections can be distinguished [15]. So, for example, IAGL processes, which are realized in atoms, should, as more accurate, be called as processes of the intra-atomic gravitational shielding (IAGS) the field of nucleus by atomic shell. This last definition we will use in article.

The gravitational field emitted by the nucleus-proton under the conditions of the IAGS realization cannot go beyond the atomic shell of protium and exists in closed oblast in the zone of nucleus. The atomic shell, in this case, is a kind of gravitational "insulator" preventing exit the field of nucleus from escaping beyond the atom. As for the FGF which emit the shell of hydrogen atom, it again, as a result of IAGS, manifests itself in external space and is not detected on the atomic nucleus.

The above-described manifestation, which is defined as the full IAGS of the 
nucleus field, is shown in Figure 2(a), which shows the areas of gravitational fields formed by sources in the composition protium atom: FGF of shell which appears beyond limit atom (indicated by straight hatching) and PGF of core (indicated in white) which, due of the IAGS effect, cannot go beyond the limits of the atomic shell. In addition, at the bottom of Figure 2(a) is shown the Earth as the third source of GP, taking part in the described gravitational influences. With the participation of the Earth, which is a powerful source of PGF is revealed all the circumstances of the gravitational scenarios related to IAGS. Since under conditions of full IAGS, the gravitational field of the nucleus cannot go beyond the atom, the gravitational interaction of the protium atom with the Earth determines exclusively the FGF emitted by the atomic shell. Between the FGF and PGF sources, i.e. between the protium atom and the Earth, the effect of gravitational levitation is realized, as a result of which these sources repel each other by the forces of positive "Dark energy" (shown in Figure 2(a) as $\boldsymbol{F}_{D E}^{+}$).

The situation with the IAGS effect, described above in relation to protium, changes significantly in the case of the hydrogen isotope-deuterium or so-called heavy hydrogen. The nucleus of deuterium, which is called deyton, consists of one proton and one neutron. It is important to note that deuton is a loosely coupled nucleus with a nucleon binding energy of just $2.22457 \mathrm{MeV}$. Unlike protium, deuterium atoms do not exhibit volatility, but are pressed against the Earth what defines the name "heavy".

The noted effect of changing the sign of the gravitational interaction of deuterium with the Earth is explained by the violation of the process of full gravitational screening of the PGF of deyton by the atomic shell. The neutron, like a proton, is a source of PGF. At inclusion of neutron in the composition of the nucleus of hydrogen atom, the intensity of PGF on the nucleus (denoted, conventionally, as $E H$ ) practically doubles. As for the shell, the value of its gravitational resistance $R_{G}$ for breakdown PGF remains the same as that of protium.

Thus, the shell that consists of single S-Graviton cannot provide complete shielding of deyton's PGF. Namely, the release (leakage) of the PGF of the nucleus beyond the atom limits leads to the compensation of the gravitational manifestation FGF of shell and is accompanied by a change in the volatility of the hydrogen atom to its so-called attraction to Earth. In this case, two opposing forces are formed between the Earth and the deuterium atomIn: repulsion of bodies by means of the $\boldsymbol{F}_{D E}^{+}$forces formed by FGF of shell and PGF of Earth and pressing them to each other by the $\boldsymbol{F}_{D E}^{-}$forces resulting from the superposition of the PGF of the Earth and the PGF of deyton which burst beyond the shell of deuterium. The resulting force $\boldsymbol{F}_{D E}$ which presses the deuterium atom to Earth is defined as the difference between the marked forces $\boldsymbol{F}_{D E}=\boldsymbol{F}_{D E}^{-}-\boldsymbol{F}_{D E}^{+}$.

A diagram of the distribution of gravitational fields emitted by the shell and the nucleus and the "DE" force effects induced by them in the case of deuterium is shown in Figure 2(b). It is important to note that the breakdown of the gravitational resistance RG of the hydrogen shell and the release of the PGF of deyton 


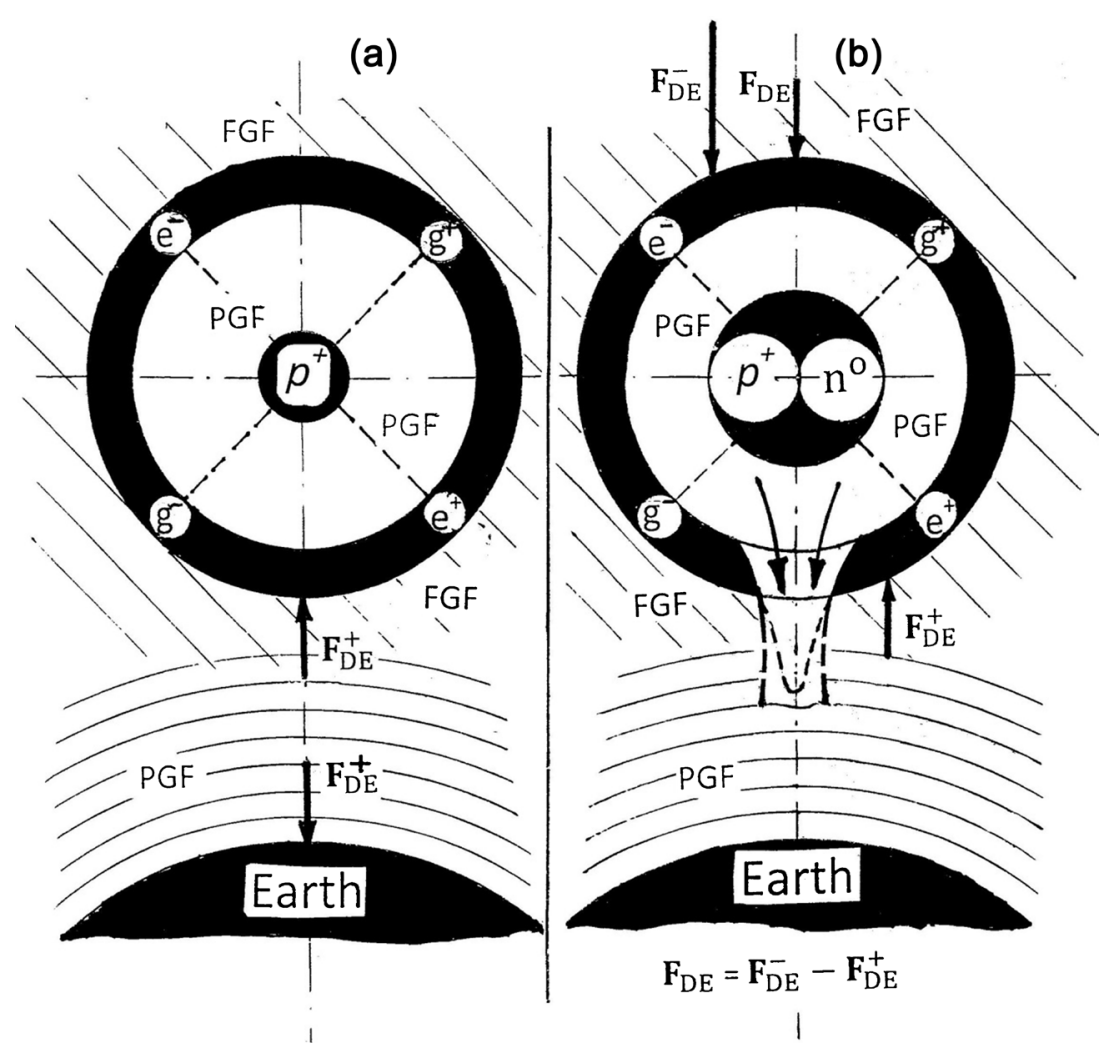

Figure 2. Schemes of distribution FGF and PGF under is conditions of realization of IAGS in the protium atom 2(a) and deuterium atom 2(b). By black color in Figure 2, stand-alone sources of GP are indicated: electromagnetic atomic shells emitting FGF and their nuclei, i.e. proton 2(a) and deyton 2(b), emitting PGF. The concentric lines indicate the Earth's PGF. The forces of the "Dark Energy" $\boldsymbol{F}_{D E}^{-}$and $\boldsymbol{F}_{D E}^{+}$, as well as their resultant force $\boldsymbol{F}_{D E}$ under the IAGS conditions of the deuterium atom, are also shown in Figure 2(a). Note: In order to simplify Figure 2(b) the diagram does not show the "DE" forces by which the deuterium atom presses the Earth to itself. These forces are antisymmetric to the forces shown in Figure 2(b) and is equal to them in magnitude.

beyond the atom limits significantly reduces the density of this field on the deuterium nucleus, which is accompanied by a decrease in the magnitude of the nuclear forces connecting the proton and neutron. It is this circumstance that explains the weak coupling of nucleons in the deuterium nucleus noted above. However, deuterium belongs to the category of stable isotopes of hydrogen.

The distribution of the PGF of deyton, shown in Figure 2(b), is in the opinion of the author, the most possible option that is realized when the IAGS effect is violated, what accompanied by the output of the PGF beyond of the atom deuterium. The intensity of the PGF of the deyton, most likely, does not greatly exceed the critical value of the intensity $\boldsymbol{E} \boldsymbol{H}_{c r}$ necessary for the breakdown of the "insulator", i.e. to overcome the gravitational resistance of the $R_{G}$ shell. Therefore, in the case of deuterium, it is most likely that only one gravitational "language" of the PGF will go beyond the atom. As for the direction of the PGF exit, it is determined by the gravitational field of the source interacting with the deuterium, i.e. of the earth. In general, as such a source can be atoms of various 
elements.

The shape and parameters of the field of PGF, which is formed outside the atom in violation of the full IAGS, require additional studies. These parameters should correspond to the necessary concentration of the nucleus field for the breakdown of shell resistance and autonomous existence beyond the atom. It is also necessary to take into account the possible processes of gravitational focusing of the PGF of the nucleus as it passes through the electromagnetic density of the shell, as well as the conditions for its interaction with the PGF of the Earth and the FGF shell beyond the deuterium atom. The dotted in Figure 2(b) is shows the possible configurations of the "language" of the PGF of the deuterium nucleus outside atomic shell.

The addition of second neutron to the nucleus of deuterium leads to the formation of a third isotope of hydrogen with the name tritium, which, as is known, is a radioactive element with a half-life of 12.32 years. The cause of radioactivity is a significant leakage of the PGF of the nucleus as a result of its release beyond the shell of tritium and the corresponding minimization of nuclear forces in its core.

According to the findings of the author the main reason for the formation of the IAGS effect is physical apartness, both of the sources and their gravitational fields in the atomic compositions. The gravitational fields emitted by the shell and the nucleons in the composition of the atom are, by their physical parameters are different gravitational fields what is determined by the differences in the values of the charges that form them. These fields cannot physically correlate in the total atomic composition, which leads to the screening by a gravitational source of an alien GP. This important circumstance is discussed in more detail below in Chapter 3.

According to the results of our study of IAGS effect in hydrogen atoms we can draw the following conclusions.

1) The effect of complete shielding of the gravitational field of the nucleus is implemented in the protium atom. In this case, the field of the proton nucleus is completely disconnected from the external gravitational manifestation of the atom, which is determined solely by the atomic shell which generates FGF.

2) The principle of shielding the gravitational field of the nucleus is determined by the physical isolation of the atomic sources of the GF, which manifests itself both in its atomic geometry and in a part of the gravitational fields emitted by them.

3) The IAGS process determines and regulates the density of the GF emitted by the nucleons in the region of the nucleus. Nuclear forces are determined by the density (intensity) of PGF on the core, i.e. the higher the density of the field, the stronger the bond between the nucleons in the nuclei. The leakage of PGF from the nucleus region, in the case of a partial removal of the gravitational "blockade", leads to a sharp weakening of the nuclear binding forces of the nucleons. This is precisely the reason for the super-weak coupling between the proton and the neutron in the deuterium nucleus, as well as the radioactivity of 
tritium.

\section{Gravitational Shielding in Atoms as Main Reason for Change in Magnitude of Nuclear Forces}

It is not difficult to foresee that the IAGS effect is a universal intra-atomic process which is implemented in the atoms of all elements. As noted above, the IAGS processes have a significant impact on the magnitude of the nuclear forces. It is important to emphasize once again that gravitational shielding is realized in the compositions of the masses (atoms, nucleons, etc.) formed by autonomous sources of gravitational fields, which differ in their physical parameters.

The magnitudes the electric and magnetic charges quarks that form the atomic shells of nucleons are more than two times smaller than the charges of the shell of atoms. The gravitational field emitted by the shells of nucleons is described by the vortex vector $\operatorname{rot}\left[\boldsymbol{E}_{n}-\boldsymbol{H}_{n}\right]$, where $\boldsymbol{E}_{n}$ and $\boldsymbol{H}_{n}$ are the electric and magnetic field strength vectors in the composition of the nucleon gravitational field. Herewith magnitudes of the marked of the vectors correspond to the conditions $E_{n}\left(H_{n}\right)<E_{a}\left(H_{a}\right)$, where the indices $n$ and a refer these quantities to the nucleon and atomic shells, respectively. Namely, the differences in the physical parameters of gravitational fields emitted by autonomous sources in the composition of atoms underlie the IAGS effects described in the article.

In the article [8], the author stated that the nuclear forces are determined primarily by gravitational forces of " $\mathrm{DE}$ ", which are realized under the influence PGF of nucleons. Since the proton and the neutron are sources of PGF, the forces of the gravitational "DE" are realized between them, which press (but do not attract) the nucleons to each other. It is these forces of "DE", according to the author, that are responsible for the formation of nuclear forces. However, the effect of IAGS, which significantly affects the situation with the magnitude of nuclear forces, was not known to the author at that time. The discovery of this effect and its introduction into basic concepts substantially complement the author's earlier assumptions regarding the nature and variability of nuclear forces.

The effect full IAGS in results in high concentration of PGF of nucleons in the region of the atomic nucleus. In the mathematical representation the distribution function of the gravitational field of free nucleon, which extends from 0 to $\infty$, convolve into function of this field in a narrow region of space from 0 to $R$, where $R$ is the radius of the nucleus. Quite roughly, such a change in the density of the gravitational field can be defined as $>10^{2}$ times. Ultrahigh density (intensity) of the PGF between nucleons in the nucleus, which is formed as a result of the full IAGS effect, accompanied by the formation of the corresponding level of negative gravitational "Dark energy" which is manifested by well-known of nuclear forces. As for the GF emitted by the atomic shell it is responsible for external processes, for example, the condensation of atoms into the composition of the substance, i.e. for the formation of a chemical bond. In other words, there is rational division of "labor" between the gravitational sources of the atom. 
The division of "labor" noted above implies an obligatory territorial isolation between the marked sources of gravitational fields in the composition of the atoms. The latter, in turn, implies the existence of forces that push these sources apart from each other for some distance and do not allow the so-called "electrons" fall to nucleus.

Everything noted above in this section was related, mainly, to the atoms of the natural stable elements that make up the periodic system of elements. At the same time, there is a huge package of atoms in whose structures the processes of screening the PGF of the nucleus are not fully performed. Such atoms are numerous isotopes of elements, on the nuclei of which, as a result of the addition of additional neutrons, supercritical values of the PGF intensity are realized. Since, in this case, the atomic shell is not able to completely shield the gravitational field of the nucleus, there is a partial release (leakage) of the field formed by the nucleons beyond the atom. As a result of this leakage, the PGF intensity at the nuclei significantly decreases compared with conditions full IAGS. The latter circumstance leads to a weakening of the nuclear forces and is manifested, for example, in such processes as the radioactivity of the nuclei.

It is important to note that the electric fields of the nucleons suspected of organizing the connection between the shell and the nucleus are most likely not directly related to the processes of gravitational shielding.

As is known, the elements in the Periodic System of Mendeleev are arranged in accordance with the increase in the size of their atomic masses or atomic weights. As for the atomic weight of any element, it is determined by the magnitude of the force of the gravitational "Dark energy" with which the atom is pressed against the Earth. It is important to note that this force is induced by a gravitational field emitted mainly by the atomic shell. In this case, as a rule, there is no contribution of the atomic nucleus to the marked "DE" force, which presses the atom to the Earth. It is this circumstance that brought about such a thing as an atomic mass defect, which is defined as the difference between the sum of the individual masses of free nucleons of a nucleus and the mass of a nucleus as a single entity. In other words, the mass of the atomic nucleus turns out to be significantly smaller than the sum of the individual masses of nucleons (protons and neutrons) in it composition. The marked decrease (defect) of the nuclear mass $\Delta m$ is determined in the existing theory by the corresponding nuclear connection energy of the nucleons $W_{n c}$ which relate as $W_{n c}=\Delta m c^{2}$. Mathematical support of the problem with the atomic mass defect can be found in textbooks on physics, as well as on Wikipedia. It is important to note that the concept of mass defect itself does not have any intelligible physical explanation, apart from its numerical definition through the value of $W_{n c}$

The discovery by the author of real magnetic charges in the structures of atoms and substance, the discovery of the electromagnetic nature of the gravitational field and the mechanism of its formation, made it possible to determine the general physical essence of the concept of atomic mass defect. Namely, the 
above-described intra-atomic shielding by the atomic shell of the gravitational fields of nucleons in the composition of atomic nuclei is the physical process that is responsible for the so-called defect of mass. Herewith the gravitational field of the nucleus, which is PGF, completely or partially, is disconnected from the so-called gravitational interaction with the Earth, which and manifests itself as the defect mass of the nucleus.

Since the gravitational "DE" is the main force factor that determines the physics of chemical bonding of atoms, chemistry, as the science of the problem of the condensation of atoms into compounds and substances, can be called gravitational chemistry. An exception here is the ionic bond, in the physics of which the participation of the electrostatic "Dark energy". However, in the processes of chemical bonding of ions, in addition to electrostatic "DE" the forces of gravitational "DE" are involved, since the ions are, as a rule, the source of PGF

There are grounds to assume that the IAGS effect is not a purely atomic effect. In his publications (see, for example, [9]), the author showed that nucleons are the small atoms, which consist of nucleon shells and nuclei. The source of GF in nucleon nuclei is the well-known positron, which is a type of mass. The positron participates in the classical gravitational interaction, i.e. shows so-called attraction to Earth. The latter implies that the positron is a source of PGF.

Since the electric and magnetic charges in the compositions of the shells of nucleons and in the structures of positrons differ significantly in magnitude, it should be assumed that the PGF emitted by marked sources in the compositions of the nucleons are different PGF. Therefore, by analogy with IAGS, one should expect the manifestation of the effect of gravitational shielding and in the structures of nucleons. This effect in the composition of nucleons should be referred to as intra-nucleon gravitational shielding (INGS). However, at the moment it is impossible to determine whether this effect is full INGS or partial.

\section{Conclusions}

Detection of the effect of IAGS described in this article turned out possible solely as result of the discovery and investigation by the author of real magnetic charges, as well as true anti-electrons in the structures of atoms and nucleons. Paradoxically, but these real particles, which make up three quarters of all real spinor particles, i.e. of charged particles in atoms and substance, more than hundred years, are not recognized by the official physical science. The introduction of the above-mentioned real particles into the basic physical concepts allowed the author to clarify, for example, such crucial questions of fundamental physics as the true nature of the gravitational field and the physical essence of mass. In addition, it is extremely important that the author managed to find out the real physics of "Dark energy" and to explain the peculiarities of its participation in the numerous effects and manifestations of the real World. In his publications it is shown that namely the forces "DE" is responsible for the dynamics and the so-called interaction of particles and masses in the Universe. So, for 
example, the gravitational "Dark energy" is the main force factor determining the Law of Universal Gravitation [16].

The main reason for ignoring all these real particles in physical science is the conditions of their retention in the structures of atoms and substances, which are fundamentally different from the retention of electrons. However, the Physics of confinement is not the only reason which blocks the path to confess these real particles by physical science. A huge negative factor on this path was Maxwell's electric magnetism (EMM), embedded in the minds of humanity in 1873 [17]. It is important to emphasize that EMM arose, exclusively, as result of superficial perception by Maxwell of famous experience Oersted's. Namely the "ingenious" guess of the Great Physicist: "electrons moving in a conductor are direct sources of the magnetic field", for more than a hundred year hindered the fruitful development of world physical science. Based on the last conclusion, the equation was written: $k \boldsymbol{J}_{e}=\operatorname{rot} \boldsymbol{H}$ known as the first Maxwell equation. The piquancy of the situation with EMM is that this "magnetism" is easily obtained in practice. It is enough to have an electric battery, conductor and a magnetic needle (compass). External simplicity and clarity formation of the magnetic field in the Oersted Experience did not suggest the need for deep thinking about its source. It was Maxwell's conclusion and his famous equation that made magnetic charges, in general, unnecessary; since and without them, using such theoretical surrogates as magnetic moments and electronic vacancies, almost everything was more or less explained.

The effect of IAGS is a universal physical effect, which necessarily manifests itself in atoms and nucleons and, therefore, concerns the physics of all varieties of mass. Physical processes such as the chemical bonding of atoms in matter, nuclear forces, and radioactivity physics are directly dependent on the IAGS effect. The article noted that such important physical concept as an atomic mass defect does not have a clear theoretical explanation. The effect of IAGS, presented in the article, allows us to quite clearly imagine which physics is behind this concept in reality. At the same time, the introduction of the IAGS effect into physical representations implies numerous revisions of existing interpretations of physical effects and even established physical laws. It is also important to note that with the use of the IAGS effect, there are ample opportunities to detect new physical effects and manifestations.

\section{Acknowledgements}

The author expresses deep gratitude to Elena Sizova and Vitaly Sizov which are for many years helped and supported author what, in many respects, contributed of the his research activities.

The author is Grateful to technical specialists: Davydov A.A., Koshelev N.V., Lomakin A.I., Moiseenkov E.V. and Staritsin S.E. which provide technical support for the main part of his experiments concerned the research of magnetic charges. 


\section{Conflicts of Interest}

The author declares no conflicts of interest regarding the publication of this paper.

\section{References}

[1] Sizov, R.A. (2001) New Representation of the Nature Magnetism, Gravity and Nuclear Forces Bonding. Akademizdat-Center "Science", Moscow, 60 p.

[2] Sizov, R.A. (2015) Journal of Modern Physics, 6, 1013-1022. https://doi.org/10.4236/jmp.2015.68106

[3] Sizov, R.A. (1971) Journal of Experimental and Theoretical Physics, 60, 1363-1370.

[4] Sizov, R.A. (2008) Electric and Magnetic Spinor Particles Are Structure-Forming Components of Mass and Electromagnetic Source Gravitation. Akademizdat-Center "Science", Moscow, 260 p.

[5] Ehrenhaft, F. (1942) Journal of the Franklin Institute, 233, 235-256. https://doi.org/10.1016/S0016-0032(42)90311-9

[6] Dirac, P.A.M. (1931) Proceedings of the Royal Society of London. Series A, 133, 60. https://doi.org/10.1098/rspa.1931.0130

[7] Sizov, R.A. (2018) Physics and Astronomy International Journal, 2, 1-5.

[8] Sizov, R.A. (2015) Journal of Modern Physics, 6, 2280-2289. https://doi.org/10.4236/jmp.2015.615232

[9] Sizov, R.A. (2018) Journal of Modern Physics, 9, 145-171. https://doi.org/10.4236/jmp.2018.92010

[10] Sizov, R.A. (2016) Journal of Modern Physics, 7, 2374-2397. https://doi.org/10.4236/jmp.2016.716206

[11] Sizov, R.A. (2015) Journal of Modern Physics, 6, 1591-1601. https://doi.org/10.4236/jmp.2015.611161

[12] Sizov, R.A. (2018) Physics and Astronomy International Journal, 2, 365-372.

[13] Sizov, R.A. (2011/2012) Matter, Antimatter and Energo-Medium Is Physical Triad of the Real World. Akademizdat-Center "Science", Moscow, 192 p.

[14] Sizov, R.A. (2016) Journal of Modern Physics, 7, 558-572. https://doi.org/10.4236/jmp.2016.76059

[15] The Gravitational Lens. (See, the Wikipedia from 13 December 2018).

[16] Sizov, R.A. (2018) Physics and Astronomy International Journal, 2, 439-443.

[17] Maxwell, J.C. (1873) Treatise on Electricity and Magnetism. Volume 1-2, Clarendon Press, Oxford. 